University of Nebraska - Lincoln DigitalCommons@University of Nebraska - Lincoln

2010

\title{
Geometrical Evaluation and Experimental Verification to Determine Representative Volume Elements of Heterogeneous Asphalt Mixtures
}

Yong-Rak Kim

University of Nebraska - Lincoln, yong-rak.kim@unl.edu

Junghun Lee

University of Nebraska - Lincoln, jlee11@unl.edu

Jamilla E. S. Lutif

University of Nebraska - Lincoln, jamilla@huskers.unl.edu

Follow this and additional works at: https://digitalcommons.unl.edu/civilengfacpub

Kim, Yong-Rak; Lee, Junghun; and Lutif, Jamilla E. S., "Geometrical Evaluation and Experimental Verification to Determine Representative Volume Elements of Heterogeneous Asphalt Mixtures" (2010). Civil Engineering Faculty Publications. 81. https://digitalcommons.unl.edu/civilengfacpub/81

This Article is brought to you for free and open access by the Civil Engineering at DigitalCommons@University of Nebraska - Lincoln. It has been accepted for inclusion in Civil Engineering Faculty Publications by an authorized administrator of DigitalCommons@University of Nebraska - Lincoln. 


\title{
Geometrical Evaluation and Experimental Verification to Determine Representative Volume Elements of Heterogeneous Asphalt Mixtures
}

\begin{abstract}
This paper presents an experimental verification of geometrically defined representative volume elements (RVEs) of heterogeneous asphalt concrete mixtures before any significant damage is initiated. A typical dense-graded Superpave mixture (12.5 mm nominal maximum aggregate size) is selected as a representative roadway paving mixture and used in this study to accomplish two parallel approaches: Geometrical analysis of mixture heterogeneity using two-dimensional actual images of asphalt concrete inner structures and experimental evaluation through uniaxial tensile tests of asphalt concrete mixtures incorporated with digital image correlation (DIC) technique. To properly address the significant heterogeneity of asphalt concrete mixtures in defining the RVE, several geometrical factors such as area fraction, gradation, orientation, and the distribution of aggregate particles in asphalt concrete mixtures are considered altogether. For the uniaxial tensile test with the DIC, the mean strains and their standard deviations captured by DIC are analyzed to confirm statistical homogeneity of RVEs evaluated from the geometrical analyses. The two approaches present similar results, indicating that typical dense-graded asphalt mixtures can be characterized for their material properties with an approximate RVE size of $60 \mathrm{~mm}$. Findings from this study further imply that the simple geometrical analysis can be an efficient tool to reasonably determine the RVE of asphalt mixtures and other granular composites where significant heterogeneity is involved.
\end{abstract}

KEYWORDS: representative volume element, asphalt mixture, digital image correlation

\section{Introduction}

Reliable material characterization and performance prediction testing of asphalt concrete mixtures has been conducted through laboratory testing of asphalt concrete specimens. The fundamental material properties resulting from tests are key inputs that are typically used as part of a model that predicts mixture performance in pavement structures. As exemplified in ASTM D3497 [1], each test designates specimen geometry and gauge length to find mixture properties. Laboratory test results from the designated specimen size and gauge length need to be representative of the global mixture characteristics and independent of the location within the mixture where measurements are taken. Therefore, identifying the proper dimension of specimen size and gauge length, typically referred to as a representative volume element (RVE), is extremely important for the characterization of mixtures. However, it is not simple due to significant geometric heterogeneity and corresponding mechanical complexity. The RVE is usually defined as the smallest but a sufficiently large volume element that can represent the effective properties of the large-scale composite [2].

Despite the significance of the RVE and the abundance of RVE studies [3-13] in the engineering field, few attempts have been made to determine appropriate RVE dimensions in asphalt mixtures [14-18]. Recently, Kim et al. [19] attempted the geometrical-

Manuscript received August 4, 2009; accepted for publication March 26, 2010; published online April 2010.

${ }^{1}$ Assistant Professor, Dept. of Civil Engineering, Univ. of Nebraska, W351 Nebraska Hall, Lincoln, NE 68588-0531. (Corresponding author), e-mail: ykim3@unl.edu

${ }^{2}$ Post-Doctoral Research Associate, Dept. of Civil Engineering, Univ. of Nebraska, W332 Nebraska Hall, Lincoln, NE 68588-0531, e-mail: jlee11@unl.edu

${ }^{3}$ Graduate Student, Dept. of Civil Engineering, Univ. of Nebraska, W329.A Nebraska Hall, Lincoln, NE 68588-0531, e-mail: jamilla@huskers.unl.edu numerical approach based on the two-dimensional image processing for determining asphalt mixture RVEs. They investigated spatial heterogeneity of mixtures by addressing statistical homogeneity of several geometrical factors such as volume fraction, gradation, orientation, and the spatial distribution of aggregate particles in three different mixtures (i.e., 9.5, 12.5 , and $19.0 \mathrm{~mm}$ nominal maximum aggregate size (NMAS)) through two-dimensional, actual images of asphalt mixture inner structures. The RVE of each mixture determined based on the geometrical criteria was then supported by finite element simulations. Three specimens of each mixture were used for the study as a preliminary-stage evaluation, and analysis results demonstrated general consistency with testing results presented in the other studies [14-18] in that the $50 \mathrm{~mm}$ gauge length seems to be appropriate for the asphalt concrete mixture where the NMAS is at or below $12.5 \mathrm{~mm}$. The study presented a clear benefit of the simple geometrical approach with much less time and effort compared to the typical extensive laboratory tests [14-18] for defining the RVE of asphalt mixtures when there is no significant damage involved in the mixture. These results imply that the mixture heterogeneity is primarily due to the initial configuration of aggregate particles.

The outcomes from the previous study [19] need further validation for a more conclusive finding. Toward this end, the $12.5 \mathrm{~mm}$ NMAS mixture was selected for this study as a typical and the most representative dense-graded Superpave mixture used today. In order to determine its proper RVE dimension, two parallel approaches were conducted; geometrical analysis of mixture heterogeneity using two-dimensional actual images of asphalt concrete inner structures and experimental evaluation by performing uniaxial tensile tests of asphalt concrete mixtures incorporated with digital image correlation (DIC). The geometrical analysis is the same approach presented in the previous study [19], but a total of ten specimens were analyzed for this study to reach a better gener- 


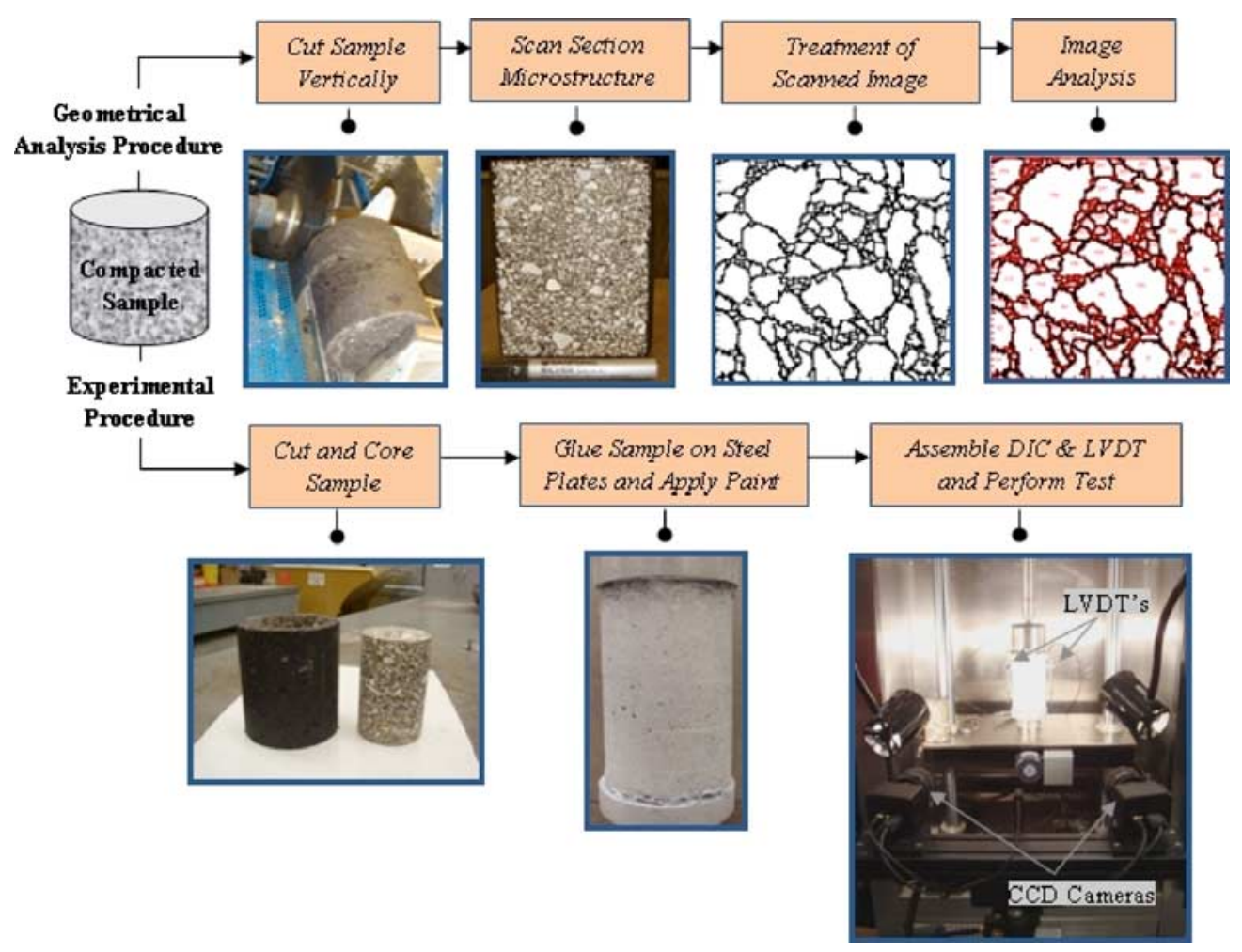

FIG. 1-Research methodology employed for this study.

ality. For the experimental evaluation, uniaxial tensile tests of two asphalt concrete cylinder specimens $(75 \mathrm{~mm}$ diameter and $140 \mathrm{~mm}$ high) were conducted with the use of high-resolution chargecoupled device (CCD) digital cameras and photogrammetric principles to measure the strain tensor on the specimen during loading. The DIC technique is nondestructive and simply a way to identify time-varying, full-field strains (or deformation) of the specimen by correlating an initial image before loading and a current image of deformed specimen at certain loading time. Since the DIC allows users to adjust gauge lengths and analysis positions after the test has been completed, the study of RVEs can be more effectively performed with much less time and effort than other conventional approaches, particularly for cases that involve significant heterogeneity. DIC test results were then compared to the geometrical analysis results so that the proper dimension of asphalt mixture RVE could be more reasonably determined.

\section{Research Objective and Scope}

The primary objective of this research is to identify a proper RVE dimension of typical dense-graded asphalt concrete mixtures where damage is not significantly involved. Since this study takes into account the spatial heterogeneity only because of aggregates in an undamaged state of asphalt concrete mixtures not involving any time-varying heterogeneities, such as cracking, the simple approach, based on the geometrical analyses of aggregate particles and the experimental DIC technique of asphalt concrete specimens, is performed and compared with the other to reach general findings. Instead of evaluating various different-type mixtures, a Superpave mixture (NMAS of $12.5 \mathrm{~mm}$ ) is selected for a more intensive analysis. The $12.5 \mathrm{~mm}$ NMAS mixture is the most frequently used today.

\section{Research Methodology}

Figure 1 illustrates the research methodology employed for this study. As mentioned earlier and presented in the figure, two parallel approaches were attempted. For each method, the Superpave mixture with a NMAS of $12.5 \mathrm{~mm}$ was compacted using a Superpave gyratory compactor to produce asphalt concrete samples (150 mm in diameter and $175 \mathrm{~mm}$ in height). The compacted sample was cut vertically using a diamond saw, and the vertical section was placed on a high-resolution scanner to capture inner structure images. The virgin inner structure image was then processed with sequential image analysis stages as proposed by Papagiannakis et al. [20], with the final image capturing only the center portion (112 $\times 112 \mathrm{~mm}^{2}$ ) of the specimen. As shown in Fig. 1, the image presents two distinct phases: white representing aggregate particles and black representing an asphalt matrix. The final image was then used to produce small subsections (referred to as trial RVEs (TRVEs)) with increasing sizes to be used for the various geometrical analyses.

For the experimental RVE evaluation based on the DIC technique, the compacted asphalt concrete samples were cored and sawn to produce cylindrical specimens $(75 \mathrm{~mm}$ in diameter and 140 $\mathrm{mm}$ tall) for the uniaxial tensile tests. Each specimen was glued to end plates that were then rigidly connected to the loading frame. For the DIC process, the specimen was painted with black and white spray until a clear contrast between white background and numerous black dots was achieved for DIC application. A closedloop servo hydraulic testing system with computer control and data acquisition was used in this study. As shown in Fig. 1, highresolution $\mathrm{CCD}$ digital cameras were placed at a predefined position, and an external light source was used to give better contrast among measuring points. Along with the DIC system, two vertical 

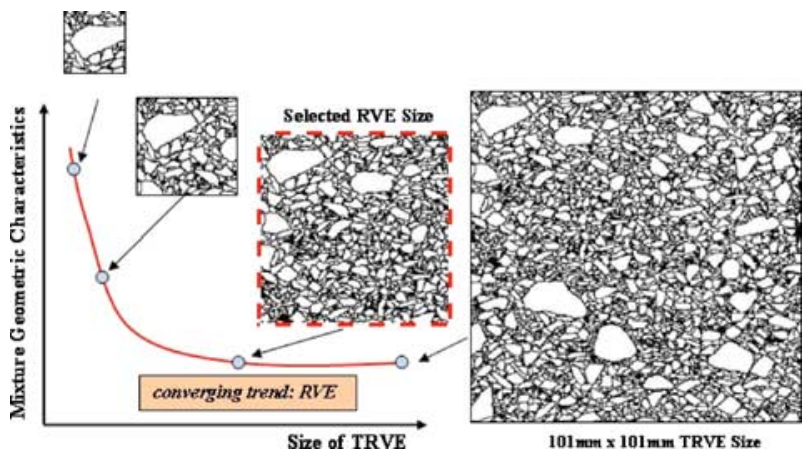

FIG. 2-A schematic illustration on the RVE evaluation based on the geometrical analysis.

linear variable differential transducers (LVDTs) were also mounted on the rear side of a specimen to measure linear deformations over the middle $100 \mathrm{~mm}$ of the specimen height when the CCD cameras captured time-varying deformation of surface images on the front side of the cylinder specimens. Figure 1 presents the DIC system (i.e., a pair of CCD digital cameras and external light sources) with typical uniaxial tensile test setup of an asphalt concrete specimen in the closed-loop servo hydraulic testing equipment.

\section{Determination of RVE Based on the Geometrical Analyses}

One of the primary requirements for an appropriate RVE is statistical homogeneity. In other words, the averaged characteristics (mechanical or geometrical) in an RVE should be statistically homogeneous so that the bulk sample characteristics and the RVE characteristics are the same. To determine the RVEs of asphalt concrete mixtures when any additional heterogeneities due to structural damage, such as cracking, is not related, Kim et al. [19] used the virgin inner structure image of mixtures to perform various geometrical analyses. Analyses results indicated that mixture heterogeneity is primarily due to the initial configuration of the aggregate particles, which further indicates that spatial homogeneity of the RVEs can be reasonably identified by investigating the statistical homogeneity of various geometric factors, such as area fraction, gradation, orientation, and the distribution of aggregate particles in asphalt concrete mixtures. The RVE of individual mixtures is there- fore identified by comparing the geometric factors obtained from several TRVE sections. The size at which all the geometric characteristics are not significantly dependent on the TRVE size, indicating convergence, is then determined as the appropriate RVE size, as illustrated in Fig. 2. Ten specimens of the $12.5 \mathrm{~mm}$ NMAS mixture were used for this study to determine the RVE dimension. For each specimen, a total of eight TRVE sections (window sizes ranging from 16.8 to $100.8 \mathrm{~mm}$ ) were generated and compared.

\section{Determination of RVE Based on the DIC Technique}

The DIC technique is a noncontact, full-field displacement/strain analysis method that compares images of deformed specimens with that of an initial undeformed specimen. With such capabilities, DIC has been used for a breadth of applications in various materials, including asphalt mixtures. Wen and Kim [21] proposed the use of DIC technique as a possible strain measurement method for asphalt mixture. Seo et al. [22,23] and Chehab et al. [24] used digital imaging techniques for mechanical testing and for calibration and validation of visco-elasto-plastic continuum damage modeling. More recently, Birgisson et al. [25] successfully used the DIC technique to monitor cracking behavior of the material at crack initiation and propagation. Based on the capabilities presented by the researchers, the DIC technique can be used to determine proper dimension of RVE for the cases with significant damage as well, since the DIC can capture real-time and continuous variations in deformation and strain of mixture components. However, for a comparison with the geometrical analysis results, this study is only interested in the evaluation of asphalt mixture RVE before any significant damage events occur.

As presented earlier, the DIC involves the use of high-resolution CCD digital cameras and photogrammetric principles to quantify displacements and corresponding strains on a specimen during loading. Two CCD cameras with a shooting angle of $25^{\circ}$ between the two capture 12 images per second, and the images are compared at many predetermined points that were marked with the black and white paint sprayed on the specimen. The points are then converted to a regular pattern that is divided into many small square areas, known as facets, typically with 5-20 pixels. The center of each facet is a measuring point. Thus, as the load is applied to the specimen, the CCD cameras capture the displacements in each facet
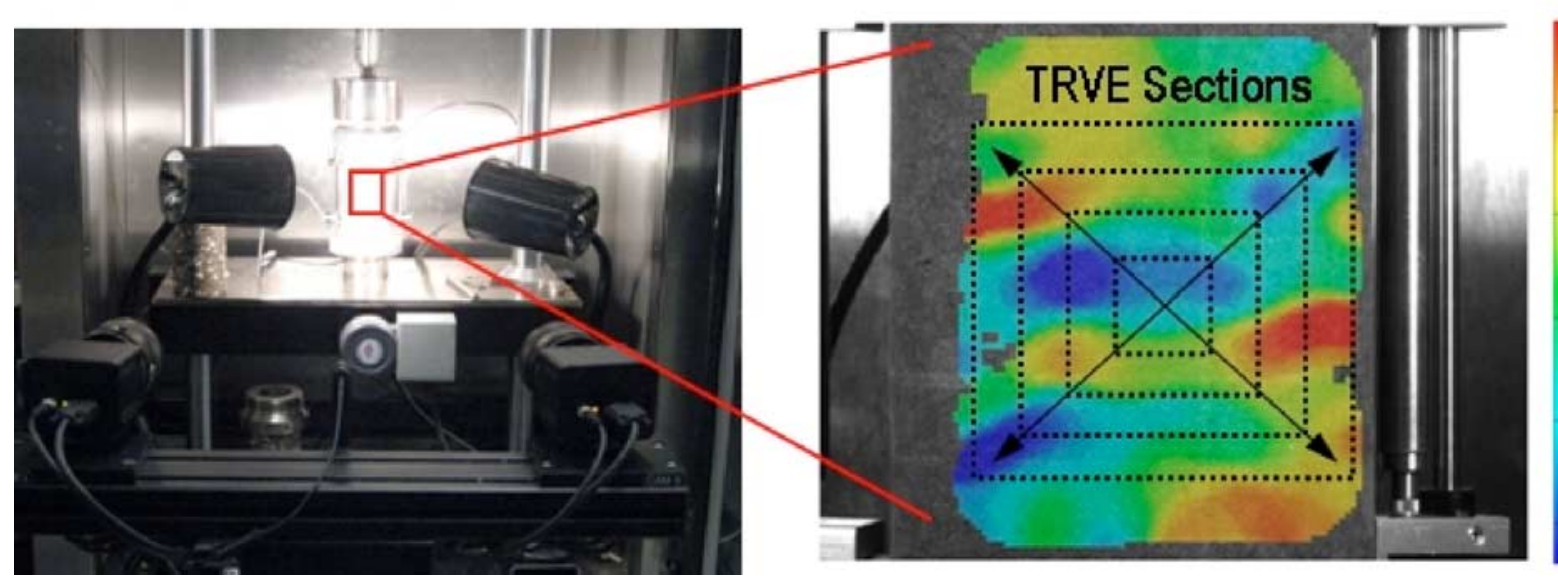


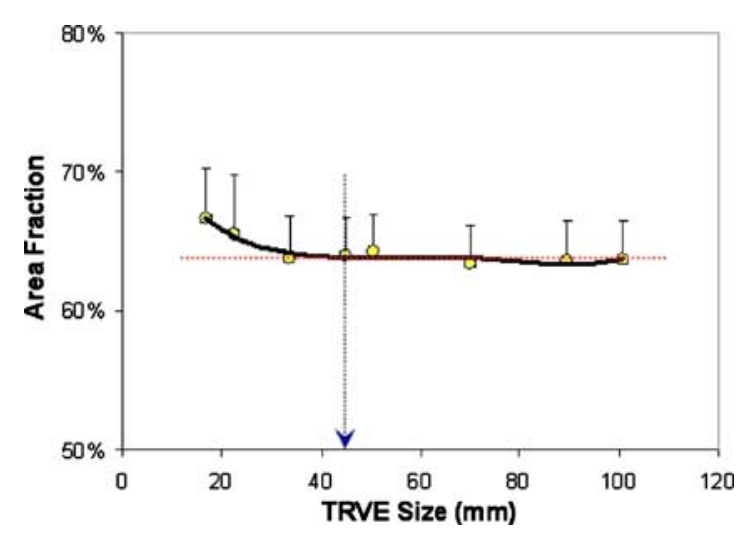

FIG. 4-Aggregate area fraction versus TRVE size.

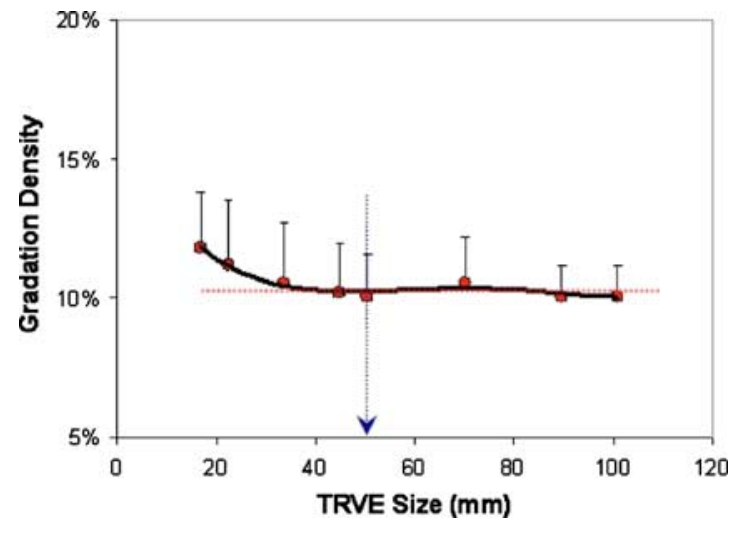

FIG. 5-Aggregate gradation density versus trve size. point, and then, by comparing the location of each facet point with its initial location from the undeformed image, the displacement vector and the strain tensor are calculated.

For the DIC testing to identify the RVE dimension, a total of seven TRVE sections (window sizes ranging from 11.2 to $70.0 \mathrm{~mm}$ ) are captured from the DIC image to compute a mean strain and a standard deviation of each TRVE section, as illustrated in Fig. 3. A strain rate of $0.001 / \mathrm{s}$ was applied to each cylindrical specimen for the uniaxial tensile testing until the mean strain of the specimen reached around 0.0003 (i.e., 300 microstrain), which is considered a level of strain where any significant nonlinear damage is not associated in the specimen. All tests were conducted at $23^{\circ} \mathrm{C}$, which is controlled by the environmental chamber. The homogenized strain (i.e., averaged strain) and its standard deviation of individual TRVE sections (11.2-70.0 $\mathrm{mm}$ ) were compared against each other to define the proper dimension of RVE as similarly conducted for the geometric analyses of mixtures. As the TRVE is closer to the RVE, variation of field variables, such as the strain considered in this study within the TRVE section and between subsequent TRVE sections, will be smaller and stabilized, which indicates that the mixture's TRVE reaches the spatial homogeneity in a statistical sense.

Along with the DIC system, two vertical LVDTs were also mounted on the rear side of the specimen to measure linear deformations over the middle of the specimen. This is to evaluate any differences and/or compatibility between two strain-measuring approaches: The conventional LVDT method often used today and the new potential method using the DIC technique before the specimens reach any significant level of nonlinear damage. Testing results from the LVDTs were compared to DIC results and are presented in the later section.

\section{Geometrical Analysis Results and Discussion}

The aggregate area fraction in each TRVE was first monitored since the area fraction is a primary variable associated with RVE in classical micromechanics [2]. Figure 4 presents the area fraction analysis results considering all ten specimens in the form of the mean area fraction and its standard deviation, which is represented by an error bar, to the mean with increasing TRVE sizes. The area fraction and variation between subsequent TRVE sections tended to decrease, but those sections larger than approximately $45 \mathrm{~mm}$ of TRVE size showed no further decrease. Based on this observation, the RVE could be defined at about $45 \mathrm{~mm}$ if the area fraction is the only factor considered to determine the RVE size.

Although the area fraction clearly provides important geometric characteristics of an asphalt mixture, it is not sufficient to identify the RVE because it cannot properly account for the size distribution of the particles in the mixture. Thus, there is a need to investigate the aggregate size distribution (i.e., gradation) in a mixture in order to monitor the existence of any particular concentration of specific particle sizes. In an attempt to account for the particle size distribution, Kim et al. [19] used gradation curves obtained from the image analysis of each TRVE, and the curves were then compared with the maximum density line to calculate the area, representing the density of each gradation curve, between the two curves. This process was attempted to quantify the gradation characteristics as a number for more efficient comparison among TRVE sections. As the TRVE size increases, the area (or gradation) variation between subsequent TRVE sections will be smaller, indicating that the TRVE is closer to the RVE. The same approach was applied to this study, and Fig. 5 presents analysis results. As can be seen in the figure, the variation of gradation density was not significantly different when the TRVE size was larger than $50 \mathrm{~mm}$.

Although the analysis of particle gradation and area fraction provides important geometric characteristics to define the RVE, spatial homogeneity might still not be guaranteed because the aggregate particles can be dispersed in the mixture in many different ways even if the area fraction and the gradation characteristics are constant. For a valid RVE, aggregates in different sizes need to be equally and randomly distributed in the whole mixture. In order to investigate the spatial homogeneity of particles, as a parallel step to the assessment of the gradation characteristics, each TRVE was subdivided into four quadrants to quantify the total number of particles and the mean area of all particles in each subquadrant to check if all quadrants show a similar number of particles and approximately the same mean area. The coefficients of variation (ratio of the standard deviation to the mean value in the quadrants) in each TRVE were calculated and compared as presented in Figs. 6 and 7. If the coefficient of variation is stable and close to zero, the difference among quadrants is insignificant. As can be seen in those figures, the coefficient of variation converged to and stabilized at around $10 \%$. Although the coefficient of variation does not reach 0 $\%$ as it should be, the converging trend implies that geometric variations among quadrants are stabilized at that level (i.e., around $10 \%$ herein), therefore a TRVE at which the converging trend starts can be considered the RVE since any sizes larger than the TRVE size present an equal level of variation. Accordingly, from Figs. 6 


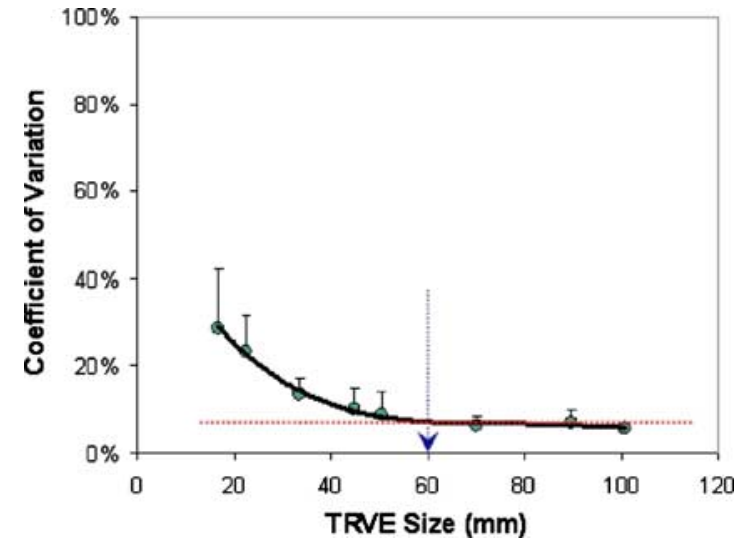

FIG. 6-Coefficient of variation among quadrants: the number of aggregate particles.

and 7, a TRVE of approximately $60 \mathrm{~mm}$ can be considered as the RVE.

The next geometric factor considered was the aggregate orientation, which was determined by measuring the angle between the major axis of each aggregate and a horizontal line on the scanned image. To quantify the aggregate orientation, the vector magnitude was calculated. The vector magnitude has been proved in numerous studies to be a useful indicator to quantify the directional measure-

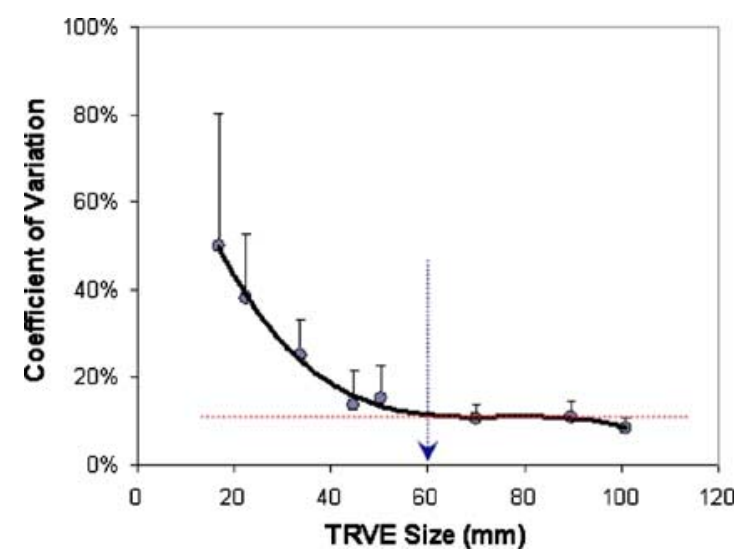

FIG. 7-Coefficient of variation among quadrants: mean area of aggregate particles.

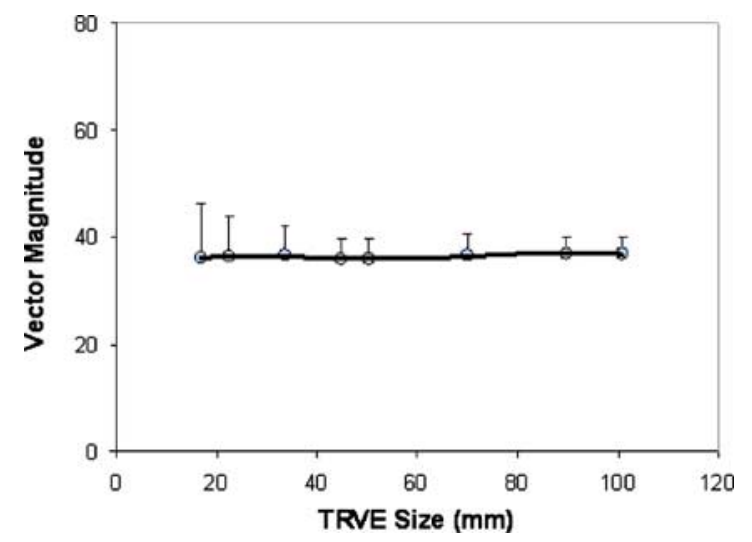

FIG. 8-Vector magnitude versus TRVE size.

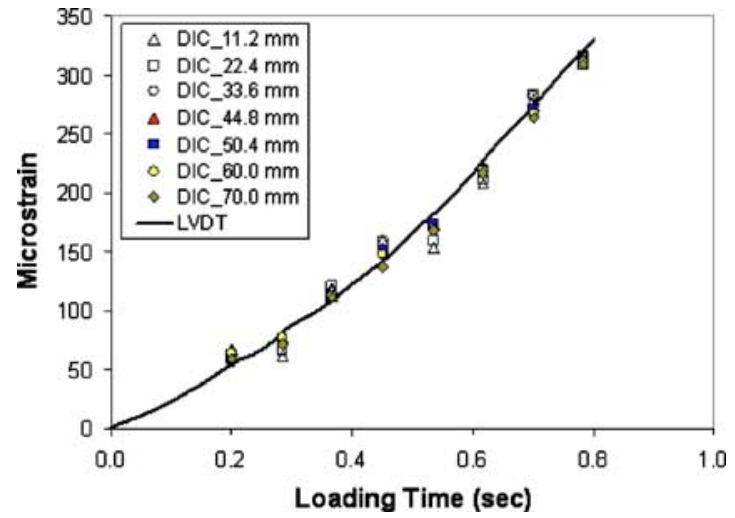

FIG. 9-Microstrain measurements: LVDTs versus DIC of each TRVE.

ments of aggregates [26,27]. The value of the vector magnitude varies from $0 \%$ to $100 \%$ : A value closer to $0 \%$ indicates that the aggregates are distributed in a more random and isotropic manner. Compared to the other geometric factors, the vector magnitude did not show clear converging trends as the TRVE size varied, as shown in Fig. 8. However, with a careful look at error bars (representing standard deviations), it can be noted that the variation of vector magnitudes tends to be stabilized when the TRVE size is greater than $45 \mathrm{~mm}$. It can be also noted that the vector magnitude of the mixture is at around $35 \%-40 \%$. This infers the existence of mixture anisotropy as previously demonstrated in other studies [26,27].

\section{DIC Analysis Results and Discussion}

Figure 9 compares time-varying strains resulting from the LVDTs and the DIC system up to around 0.0003 strain (i.e., 300 microstrain). As seen, there is a good match between the LVDT measurement (from $10 \mathrm{~cm}$ gauge length in the middle of specimen) and the DIC results at any size of TRVE sections. From the result, it can be inferred that linear strains from the LVDTs are quite compatible with strain measurements obtained from the specimen surface when the testing is conducted to asphalt mixtures before any significant damage-related events occur.

Figure 10 presents homogenized (averaged) vertical strains selected at two loading times along the loading direction as the TRVE size varies. The mean strain did not show any significant changes as the TRVE size increased. This was true of all specimens repeated for this study. However, a different plot relating the standard devia-

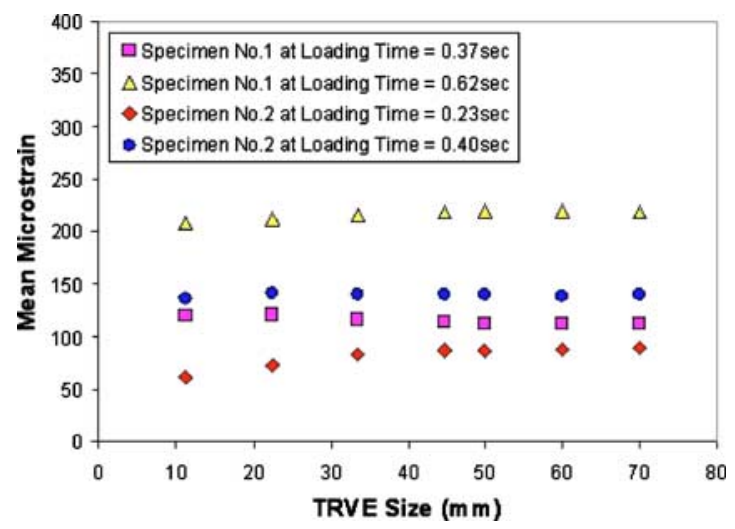

FIG. 10-Mean microstrain versus TRVE size. 


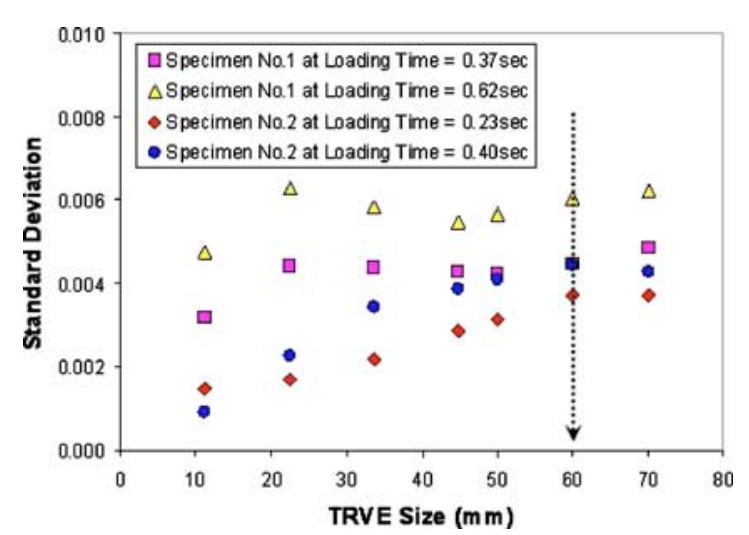

FIG. 11-Standard deviation of microstrains versus TRVE size.

tion of strains at each TRVE section to the size of TRVEs presented an increasing and converging trend as shown in Fig. 11. The increasing trend with larger TRVEs presented in the figure seems reasonable because the mixture consists of two distinct phases (i.e., aggregate particles and asphalt matrix) where strains between two phases are considerably different. Strains of asphalt phase are much greater than strains from aggregate particles, which consequently implies that the variance of strains in a TRVE becomes larger if the area fraction of particles in the TRVE is smaller, which is the case observed in Fig. 4. When the increasing trend stops and stabilizes, the TRVE is close to the RVE because the stabilized standard deviation means spatial homogeneity of two phases in the mixture. In this study, the standard deviation converged for a TRVE size larger than approximately $60 \mathrm{~mm}$.

The experimental DIC test results are generally in good agreement with the findings from the geometrical RVE analyses. $\mathrm{Nu}-$ merical finite element analyses conducted in the previous study [19] as well as other experimental results obtained by researchers [14-18] are generally consistent with outcomes from this study in that the 50-60 mm gauge length seems to be appropriate for the asphalt concrete mixtures where the nominal maximum aggregate size is at or below $12.5 \mathrm{~mm}$.

With significant damage and/or larger aggregate sizes involved in the mixture, the study of RVE should be performed with more care since the mixture might be subjected to additional heterogeneities, such as cracks, localized yielding, and possibly aggregate movements. When the mixture is subjected to significant damage, the DIC technique presented in this paper can be a particularly useful tool since the DIC captures real-time, continuous, twodimensional variations of mixture components. The authors have performed the study of RVEs with damage as a follow-up of this.

\section{Significance and Uses of Research Findings}

The good agreement between geometric analyses and various experimental approaches suggests that the simple geometric method based on the image analysis and basic statistical analyses of mixture heterogeneity can be used as an efficient tool to define the RVE of asphaltic composites instead of repeating time-consuming and expensive mechanical tests when the mixture is not strongly associated with nonlinear damage. The similar approach can be applied to various types of heterogeneous mixtures.

Another direct benefit of this study is related to the mechanistic modeling of asphalt concrete mixtures. One of approaches cur- rently receiving increased attention from the asphalt materials and pavement mechanics community is computational micromechanics modeling, which is probably the best way to account for the effects of individual mixture constituents and their interactions on the overall behavior of mixtures with regard to heterogeneity, anisotropy, nonlinear inelasticity, and even damage growth. To realize the micromechanics modeling, appropriate RVEs must be identified because a micromechanics model requires the effective properties of a heterogeneous bulk mixture obtained by a homogenization process dealing with a heterogeneous RVE. The beauty of micromechanics is therefore highlighted through the concept of RVEs. Once the RVE has been determined, it can simply be reconstructed for the computational simulations that can virtually predict mixture properties and performance, not relying on the timeconsuming, expensive mixture-level laboratory tests.

\section{Summary and Conclusions}

Based on this study, the key findings and related conclusions are summarized as follows:

- Various geometrical factors such as area fraction, gradation, orientation, and the distribution of aggregate particles in asphalt concrete mixtures were considered to determine the proper dimension of RVE for a typical dense-graded Superpave mixture (12.5 mm NMAS mixture). Analysis results from various geometrical factors altogether showed that effective properties of the mixture can be characterized with an approximate RVE size of $60 \mathrm{~mm}$. This, in turn, indicates that current mixture property tests such as the dynamic modulus test [1] and the low-temperature creep compliance test [28] are generally reasonable to identify mixture properties.

- The geometrically defined RVE for the $12.5 \mathrm{~mm}$ NMAS mixture was verified by performing the constant displacement rate tensile tests of asphalt concrete specimens incorporated with the DIC system. As a result, the TRVE size of around $60.0 \mathrm{~mm}$ was found as a reasonable RVE, since standard deviation of strains stabilized at around $60.0 \mathrm{~mm}$ TRVE size, which indicates the spatial homogeneity.

- The geometrical and experimental results from this study are generally consistent with finite element method results shown in the previous study and in other conventional studies mostly based on experimental tests with different specimen geometries. The good agreement between geometrical analyses and various experimental approaches suggests that the simple geometric method can be useful to define the RVE of asphaltic composites and other mixtures instead of repeating the time-consuming and expensive mechanical tests when the mixture is not associated with significant nonlinear damage.

- The outcomes of this study can be extended to RVE study when the mixture is subjected to significant damage. In particular, the DIC technique can be recommended as a potentially efficient tool for defining the RVE of asphalt mixtures with additional heterogeneities such as cracks. Furthermore, the RVEs determined from this and follow-up studies can be used for advanced computational micromechanics simulations that can virtually predict mixture properties and performance without having to rely on the expensive, timeconsuming mixture-level tests. 


\section{Acknowledgments}

The authors gratefully acknowledge the National Science Foundation (Grant No. CMMI-0644618) and the Texas A\&M Research Foundation for their financial support.

\section{References}

[1] ASTM D-3497, 2003, "Standard Test Method for Dynamic Modulus of Asphalt Mixtures," Annual Book of ASTM Standards, ASTM International, West Conshohocken, PA.

[2] Hashin, Z., "Analysis of Composite Materials. A survey," J. Appl. Mech., Vol. 50, 1983, pp. 481-505.

[3] Hill, R., "Elastic Properties of Reinforced Solids: Some Theoretical Principles," J. Mech. Phys. Solids, Vol. 11, 1963, pp. 357-372.

[4] Drugan, W. J. and Willis, J. R., "A Micromechanics-based Non-local Constitutive Equation and Estimates of Representative Volume Element Size for Elastic Composites," J. Mech. Phys. Solids, Vol. 44, 1996, pp. 497-524.

[5] Gusev, A. A., "Representative Volume Element Size for Elastic Composites: A Numerical Study," J. Mech. Phys. Solids, Vol. 45, 1997, pp. 1449-1459.

[6] Kanit, T., Forest, S., Galliet, I., Mounoury, V., and Jeulin, D., "Determination of the Size of the Representative Volume Element for Random Composites: Statistical and Numerical Approach,” Int. J. Solids Struct., Vol. 40, 2003, pp. 3647-3679.

[7] Liu, C., "On the Minimum Size of Representative Volume Element: An Experimental Investigation,” Exp. Mech., Vol. 45, 2005, pp. 238-243.

[8] Helms, K., Allen, D. H., and Hurtado, L., “A Model for Predicting Grain Boundary Cracking in Polycrystalline Viscoplastic Materials Including Scale Effects," Int. J. Fract., Vol. 95, 1999, pp. 175-194.

[9] Ostoja-Starzewski, M., "Microstructural Randomness versus Representative Volume Element in Thermomechanics," $J$. Appl. Mech., Vol. 69, 2002, pp. 25-35.

[10] Swaminathan, S., Ghosh, S., and Pagano, N. J., "Statistically Equivalent Representative Volume Elements for Unidirectional Composite Microstructures: Part I-Without Damage," J. Compos. Mater., Vol. 40, 2005, pp. 583-604.

[11] Swaminathan, S. and Ghosh, S., "Statistically Equivalent Representative Volume Elements for Unidirectional Composite Microstructures: Part II-with Interfacial Debonding," J. Compos. Mater., Vol. 40, 2005, pp. 605-621.

[12] Terada, K., Hori, M., Kyoya, T., and Kikuchi, N., "Simulation of the Multi-Scale Convergence in Computational Homogenization Approaches," Int. J. Solids Struct., Vol. 37, 2000, pp. 2285-2311.

[13] van der Sluis, O., Schreurs, P. J. G., Brekelmans, W. A. M., and Meijer, H. E. H., "Overall Behaviour of Heterogeneous Elastoviscoplastic Materials: Effect of Microstructural Modelling," Mech. Mater., Vol. 32, 2000, pp. 449-462.

[14] Romero, P. and Masad, E., "Relationship between the Representative Volume Element and Mechanical Properties of Asphalt Concrete," J. Mater. Civ. Eng., Vol. 13, 2001, pp. 77-84.

[15] Weissman, A. L., Harvey, J., Sackman, J. L., and Long, F., "Selection of Laboratory Test Specimen Dimension for Per- manent Deformation of Asphalt Concrete Pavements," Transportation Research Record. 1681, Transportation Research Board, Washington, D.C., 1999, pp. 113-120.

[16] Harvey, J., Guada, I., and Long, F., "Effects of Material Properties, Specimen Geometry, and Specimen Preparation Variables on Asphalt Concrete Tests for Rutting," Electron. J. Assoc. Asph. Paving Technol., Vol. 69, 2000, pp. 236-280.

[17] Witczak, M. W., Bonaquist, R., Von Quintus, H., and Kaloush, K., "Specimen Geometry and Aggregate Size Effects in Uniaxial Compression and Constant Height Shear Tests," Electron. J. Assoc. Asph. Paving Technol., Vol. 69, 2000, pp. 733-793.

[18] Chehab, G. R., O’Quinn, E., and Kim, Y. R., "Specimen Geometry Study for Direct Tension Test Based on Mechanical Tests and Air Void Variation in Asphalt Concrete Specimens Compacted by Superpave Gyratory Compactor," Transportation Research Record. 1723, Transportation Research Board, Washington, D.C., 2000, pp. 125-132.

[19] Kim, Y., Lutif, J. E. S., and Allen, D. H., "Determining Representative Volume Elements of Asphalt Concrete Mixtures Without Damage," Transportation Research Record. 2127, Transportation Research Board, Washington, D.C., 2009, pp. 52-59.

[20] Papagiannakis, A. T., Abbas, A., and Masad, E., "Micromechanical Analysis of Viscoelastic Properties of Asphalt Concretes," Transportation Research Record. 1789, Transportation Research Board, Washington, D.C., 2002, pp. 113-120.

[21] Wen, H. and Kim, Y. R., "Simple Performance Test for Fatigue Cracking and Validation with WesTrack Mixtures," Transportation Research Record. 1789, Transportation Research Board, Washington, D.C., 2002, pp. 66-72.

[22] Seo, Y., Kim, Y. R., Witczak, M. W., and Bonaquist, R., “Application of Digital Image Correlation Method to Mechanical Testing of Asphalt-Aggregate Mixtures," Transportation Research Record. 1789, Transportation Research Board, Washington, D.C., 2002, pp. 162-172.

[23] Seo, Y., Kim, Y. R., Schapery, R. A., Witczak, M. W., and Bonaquist, R., "A Study of Crack-Tip Deformation and Crack Growth in Asphalt Concrete Using Fracture Mechanics," Electron. J. Assoc. Asph. Paving Technol., Vol. 73, 2004, pp. 697-730.

[24] Chehab, G. R., Seo, Y., and Kim, Y. R., "Viscoelastoplastic Damage Characterization of Asphalt-Aggregate Mixtures Using Digital Image Correlation,” Int. J. Geomech., Vol. 7, 2007, pp. 111-118.

[25] Birgisson, B., Montepara, A., Romeo, E., Roque, R., Roncellla, R., and Tebaldi, G., "Determination of Fundamental Tensile Failure Limits of Mixtures," Electron. J. Assoc. Asph. Paving Technol., Vol. 76, 2007, pp. 303-344.

[26] Masad, E., Muhunthan, V., Shashidhar, N., and Harman, T., "Internal Structure Characterization of Asphalt Concrete Using Image Analysis," J. Comput. Civ. Eng., Vol. 13, 1999, pp. 88-95.

[27] Muhunthan, B., Masad, E., and Assaad, A., "Measurement of Uniformity and Anisotropy in Granular Materials," Geotech. Test. J., Vol. 23, 2000, pp. 423-431.

[28] AASHTO T322, 2007, "Standard Method of Test for Determining the Creep Compliance and Strength of Hot-Mix Asphalt (HMA), Using the Indirect Tensile Test Device." 